\title{
REVISORES 2014
}

EI Equipo Editorial desea agradecer, muy sinceramente, el trabajo realizado por los revisores. Somos conscientes del esfuerzo que ello supone.

Ahmed, Wondimu. University of Groningen (Países Bajos)

Alegre, Albert. East Stroudsburg University (EEUU)

Álvarez, Ibis. Universidad Autónoma d Barcelona (España)

AlQaryouti, Ibrahim. Sultan Qaboos University (Omán)

Ainscow, Mel . University of Manchester (Reino Unido)

Assouline, SEEUUn G. University of Iowa (EEUU)

Barraza, Arturo. Universidad Pedagógica de Duranego (México)

Bartels, Jared. University Central of Missoury (EEUU)

Bembenutty, Heffer. Queen College of New Yoyk (EEUU)

Benitez, Juan Luis. Universidad de Granada (España)

Bred, Betty. Nord West University (Sudáfrica)

Calero, Maria Dolores. Universidad de Granada (España)

Cardelle-Elawar, María. Arizona State University (EEUU)

Castejón, Juan Luis. Universidad de Alicante (España)

Cerezo, Fuensanta. Universidad de Murcia (España)

Clariana, Mercè. Universidad Autónoma de Barcelona (España)

Dejonckheere, Peter. University College of Katho Tielt (Bélgica)

de la Fuente, Jesús. University of Almería (España)

Dilmark, Bülent. Sekul University (Turquía)

Elizalde, Alejandra. Universidad de las Américas (México)

Eren, Altay. Gölköy Campus, Bolu, (Turquía)

Enriques, Manuel Joaquim. University of Lisbon (Portugal)

Fawcett, Angela. University of Sheffield (Reino Unido) 
Fernández, Antonio. Universidad de Granada (España)

Flores, Raquel. Universitat Jaume I (España)

Font, Vicenç. Universidad de Barcelona (España)

Gadelrab, Hesham F., Umm Alqra University, Makahh Almokrama (Arabia Saudí)

García-López, María del Mar. Universidad de Almería (España)

Godino, Juan D. Universidad de Granada (España)

Gustems, Josep. Universidad de Barcelona (España)

Hilpert, Jonathan C. Georgia Southern University (EEUU)

Hinz, Arnold. University of Education Ludwigsburg (Alemania)

Inglés, Cándido J. Universidad Miguel Hernández, Elche (España)

Ivet Chávez, Blanca . Universidad Nacional Autónoma de México (México)

Jara, Marisol. Hospital Psiquiatrico (Costa Rica)

Gil, Nuria. Universidad de Extremadura (España)

Lee, Joon Sun. Hunter College, The City University of New York (EEUU)

Linderholm, Tracy. Georgia Southern University (EEUU)

Longobardi, Claudio. University of Turin (Italia)

López, Magdalena. Universidad Pontificia de Buenos Aires (Argentina)

McLaughlin, T. F. Gonzaga University, Spokane, Washington (EEUU)

Mcloughling, Caven S. Kent State University, Ohio (EEUU)

Marugán, Montserrat.Universidad de Valladolid (España)

Martín, Isabel. Universidad de Sevilla (España)

Martínez- Arias, Rosario. Universidad Complutense de Madrid (España)

Martínez-Baena, Alejandro César. Universidad de Granada (España)

Martínez-Vicente, José Manuel. Universidad de Almería (España)

Moriana, Juan Antonio. Universidad de Córdoba (España)

Muñiz, José. Universidad de Oviedo (España)

Näsström, Gunilla . Umeå University (Suecia)

Núñez, $M^{a}$ Cristina. Universidad Complutense de Madrid (España)

Olani, Aboma. University of Groningen (Países Bajos)

Palomera, Raquel. Universidad de Cantabria (España)

Pérez-Escoda, Nuria. Universidad de Barcelona (España)

Phan, Huy P. The University of New England (Australia)

Ponce, Hector. EEUUCH (Chile)

Rita Paoloni, Paola Verónica. Universidad de Córdoba (Argentina)

Roebken,U. Heinke niversity of Oldenburg (Alemania) 
Rocabert, Esperanza. Universidad de Valencia (España)

Rucillo, María Teresa. Universidad de Jaén (España)

Sander, Paul. UWIC, Cardiff Metropolitan University (Reino Unido)

Segovia, Isidoro. Universidad de Granada (España)

Tejeiro, Ricardo A. School of Psychology, University of Liverpool (Reino Unido)

Trianes, María Victoria. Universidad de Málaga (España)

Was, Cristopher . Kent State University, Ohio (EEUU)

White, Maria C. Nyack College, New York (EEUU)

Witt, Marcuss. Marcuss University of Bath (Reino Unido)

Zavala, María Alicia. Universidad de Guadalajara (México)

\section{Porcentaje de aceptación-rechazo (2014):}

- Recibidos: $136(100 \%)$

- Rechazados: 95 (70\%)

- Aceptados: 41 (30\%) 


\section{[Página en blanco por razones de paginación]}

